\title{
Candidaspongiolides, Distinctive Analogs of Tedanolide from Sponges of the Genus Candidaspongia
}

Tamara L. Meragelman, Richard H. Willis, Girma M. Woldemichael, Andrew Heaton, Peter T. Murphy, Kenneth M. Snader, David J. Newman, Rob van Soest, Michael R. Boyd, John H. Cardellina II, and Tawnya C. McKee*

\section{Supporting Information}

Table S1. Comparison of ${ }^{1} \mathrm{H}$ NMR Assignments ( $500 \mathrm{MHz}, \mathrm{CDCl}_{3}$ ) for tedanolide (orig. and synthetic) and candidaspongiolide mixture (3)

Figure S 1. ${ }^{1} \mathrm{H}$ NMR spectrum of candidaspongiolides (3) in $\mathrm{CDCl}_{3}$

Figure S 2. ${ }^{1} \mathrm{H}$ NMR spectrum of candidaspongiolides (3) in acetone- $d_{6}$

Figure S 3. ${ }^{13} \mathrm{C}$ NMR spectrum of candidaspongiolides (3) in acetone- $d_{6}$

Figure S 4. COSY spectrum of candidaspongiolides (3) in acetone- $d_{6}$

Figure S 5. HSQC spectrum of candidaspongiolides (3) in acetone- $d_{6}$

Figure S 6. ${ }^{1} \mathrm{H}$ NMR spectrum of candidaspongiolide core (4) in acetone- $d_{6}$

Figure $S$ 7. COSY spectrum of candidaspongiolide core (4) in acetone- $d_{6}$

Figure S 8. HSQC spectrum of candidaspongiolide core (4) in acetone- $d_{6}$

Figure $\mathrm{S} 9$. $\mathrm{HMBC}(4 \mathrm{~Hz})$ spectrum of candidaspongiolide core (4) in acetone- $d_{6}$

Figure S 10. HMBC ( $8 \mathrm{~Hz})$ spectrum of candidaspongiolide core (4) in acetone- $d_{6}$

Figure S 11. NOESY spectrum of candidaspongiolide core (4) in acetone- $d_{6}$ 
Table S1. Comparsion of ${ }^{1} \mathrm{H}$ NMR Assignments (500 MHz, $\mathrm{CDCl}_{3}$ ) for Tedanolide (orig. ${ }^{5}$ and synthetic ${ }^{7}$ and Candidaspongiolide mixture (3)

\begin{tabular}{|c|c|c|c|}
\hline position & $\begin{array}{c}\text { Candidaspongiolide (3) } \\
\delta_{\mathrm{H}} \text { mult, }(\mathrm{J}=\mathrm{Hz}) \\
\end{array}$ & $\begin{array}{c}\text { Tedanolide (1, Natural Prod.) } \\
\delta_{\mathrm{H}} \text { mult, }(\mathrm{J}=\mathrm{Hz})\end{array}$ & $\begin{array}{c}\text { Tedanolide (synthetic) } \\
\delta_{\mathrm{H}} \text { mult, }(\mathrm{J}=\mathrm{Hz})\end{array}$ \\
\hline \multirow[t]{2}{*}{2} & $3.96 \mathrm{dd}(7.3,1.0)$ & $3.87 \mathrm{dd}(8.7,1.7)$ & $3.89, \mathrm{dd}(7.9,0.7)$ \\
\hline & $\mathrm{OH} 2.85 \mathrm{~d}(7.3)$ & $\mathrm{OH} 2.83 \mathrm{~d}(8.7)$ & $\mathrm{OH} 2.76 \mathrm{~d}(8.5)$ \\
\hline 3 & $3.64 \mathrm{dd}(7.8,1.0)$ & $3.68 \mathrm{dd}(8.6,1.7)$ & $3.68 \mathrm{dd}(8.5,1.4)$ \\
\hline 4 & $3.12 \mathrm{~m}$ & $3.25 \mathrm{dq}(8.6,7.2)$ & $3.36-3.28 \mathrm{~m}$ \\
\hline 6 & $3.18 \mathrm{dq}(10.7,7.3)$ & $3.04 \mathrm{dq}(9.9,6.8)$ & $3.07-3.00 \mathrm{~m}$ \\
\hline \multirow[t]{2}{*}{7} & $5.39 \mathrm{~d}(10.7)$ & $4.11 \mathrm{dd}(9.9,2.5)$ & $4.11 \mathrm{dd}(7.5,1.0)$ \\
\hline & & 7-OH 1.56 d (2.5) & \\
\hline 9 & 5.60 br d (9.3) & $5.47 \mathrm{dq}(10.8,1.4)$ & $5.47 \mathrm{dd}(8.5,1.0)$ \\
\hline 10 & $3.38 \mathrm{dq}(9.3,6.8)$ & $3.42 \mathrm{dq}(10.8,6.6)$ & $3.44-3.37 \mathrm{~m}$ \\
\hline \multirow[t]{2}{*}{12} & a $2.66 \mathrm{dd}(16.1,9.8)$ & $2.53 \mathrm{dd}(16.9,3.8)$ & $2.47 \mathrm{dd}(17.0,3.1)$ \\
\hline & b 2.47 br dd $(16.1,2.4)$ & $2.58 \mathrm{dd}(16.9,9.0)$ & $2.57 \mathrm{dd}(16.7,9.2)$ \\
\hline \multirow[t]{2}{*}{13} & $4.40 \mathrm{~m}$ & 4.31 dddd $(9.0,6.5,3.3,3.2)$ & $4.31 \mathrm{~m}$ \\
\hline & $\mathrm{OH} 3.45 \mathrm{~d}(4.9)$ & $\mathrm{OH} 3.37 \mathrm{~d}(3.2)$ & OH $3.44-3.37 \mathrm{~m}$ \\
\hline 14 & $\mathrm{OH} 3.31 \mathrm{br} \mathrm{s}$ & $3.031 \mathrm{H} \mathrm{dq}(6.5,6.6)$ & $3.07-3.001 \mathrm{H} \mathrm{m}$ \\
\hline 16 & $4.02 \mathrm{dt}(10.9,3.7)$ & 3.54 ddd $(11.6,9.5,4.1)$ & $3.54 \mathrm{ddd}(11.6,9.4,3.9)$ \\
\hline \multirow[t]{2}{*}{17} & $3.12 \mathrm{~m}$ & $3.24 \mathrm{dd}(9.5,3.9)$ & $3.24 \mathrm{dd}(9.4,3.6)$ \\
\hline & $\mathrm{OH} 2.77$ br d (2.9) & $\mathrm{OH} 2.20 \mathrm{~d}(3.6)$ & $\mathrm{OH} 2.14 \mathrm{~d}(4.1)$ \\
\hline 19 & $2.56 \mathrm{~d}(9.3)$ & $2.65 \mathrm{~d}(9.4)$ & $2.61 \mathrm{~d}(9.2)$ \\
\hline 20 & $2.44 \mathrm{~m}$ & $2.45 \mathrm{ddq}(10.8,9.4,6.9)$ & $2.48-2.40 \mathrm{~m}$ \\
\hline 21 & $5.23 \mathrm{dt}(10.7,1.5)$ & $5.24 \mathrm{ddq}(10.8,10.8,1.7)$ & $5.23 \mathrm{ddq}(10.6,10.4,1.7)$ \\
\hline 22 & $5.48 \mathrm{dq}(10.7,6.8)$ & $5.46 \mathrm{dq}(10.8,7.4)$ & $5.46 \mathrm{dq}(10.8,6.8)$ \\
\hline 23 & $1.59 \mathrm{dd}(6.8,1.5)$ & $1.61 \mathrm{dd}(7.4,1.7)$ & $1.61 \mathrm{dd}(8.2,1.4)$ \\
\hline 24 & $1.18 \mathrm{~d}(6.8)$ & $1.24 \mathrm{~d}(7.2)$ & $1.23 \mathrm{~d}(7.2)$ \\
\hline 25 & $1.13 \mathrm{~d}(7.3)$ & $1.29 \mathrm{~d}(6.8)$ & $1.28 \mathrm{~d}(7.2)$ \\
\hline 26 & 1.54 br d (1.0) & $1.63 \mathrm{~d}(1.4)$ & $1.62 \mathrm{~d}(1.4)$ \\
\hline 27 & $1.07 \mathrm{~d}(6.8)$ & $1.12 \mathrm{~d}(6.5)$ & $1.12 \mathrm{~d}(6.5)$ \\
\hline \multirow[t]{2}{*}{28} & a $4.441 \mathrm{H} \mathrm{d}(11.7)$ & $1.123 \mathrm{H} \mathrm{d}(6.5)$ & $1.113 \mathrm{H} \mathrm{d}(7.2)$ \\
\hline & b $4.191 \mathrm{H} \mathrm{d}(11.7)$ & & \\
\hline
\end{tabular}




\begin{tabular}{llll}
29 & a $4.10 \mathrm{dd}(10.2,10.9)$ & $4.11 \mathrm{dd}(11.6,11.6)$ & $4.12 \mathrm{dd}(11.1,11.1)$ \\
& b $4.17 \mathrm{dd}(9.8,3.7)$ & $4.26 \mathrm{dd}(11.6,4.1)$ & $4.26 \mathrm{dd}(10.6,4.1)$ \\
30 & $1.38 \mathrm{~s}$ & $1.39 \mathrm{~s}$ & $1.39 \mathrm{~s}$ \\
31 & $1.11 \mathrm{~d}(6.4)$ & $1.10 \mathrm{dd}(6.7,1.7)$ & $1.09 \mathrm{~d}(7.2)$ \\
32 & $3.28 \mathrm{~s}$ & $3.30 \mathrm{~s}$ & $3.30 \mathrm{~s}$ \\
\hline
\end{tabular}




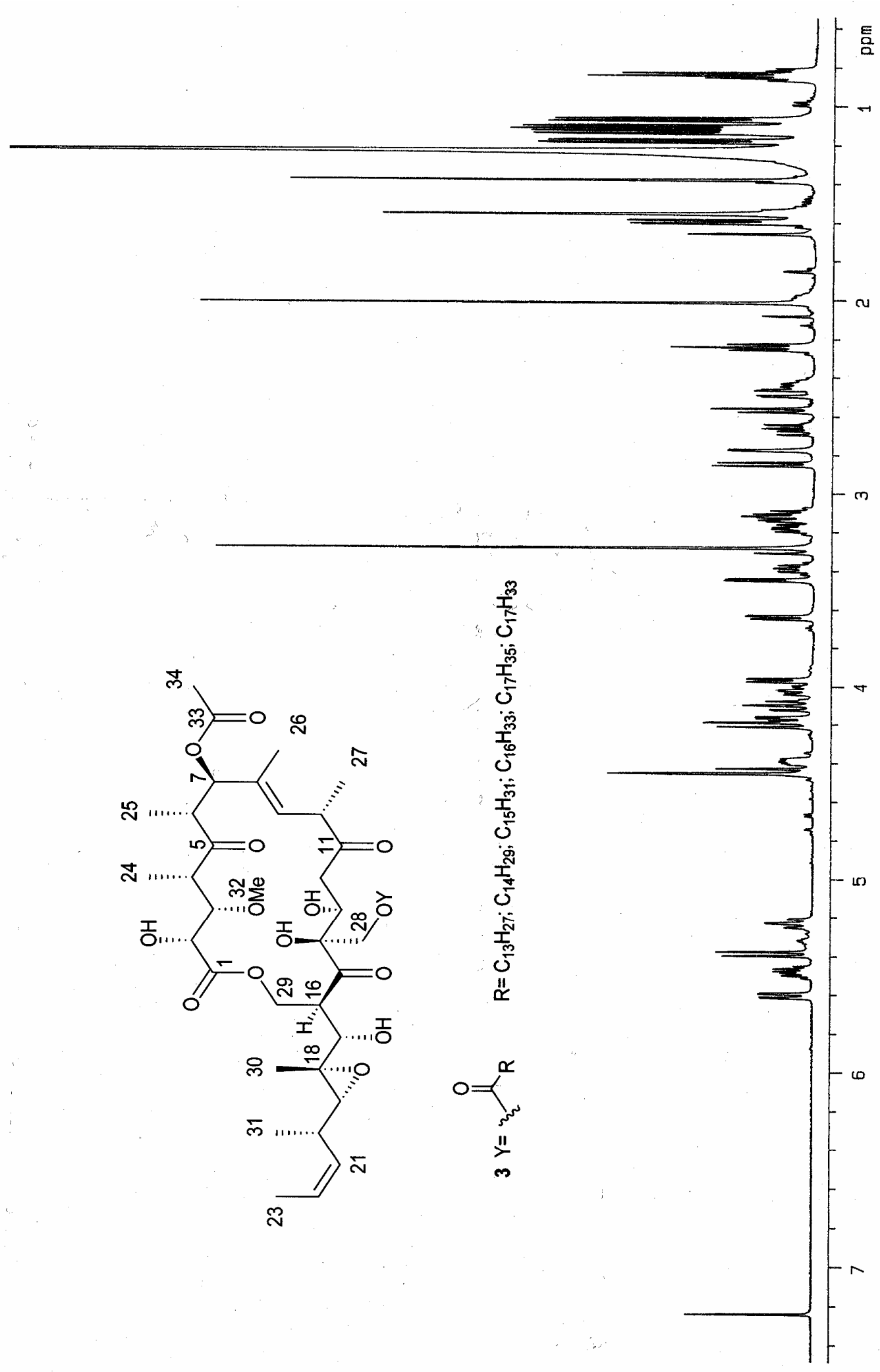

Figure S 1. ${ }^{1} \mathrm{H}$ NMR spectrum candidaspongiolides (3) in $\mathrm{CDCl}_{3}$ 


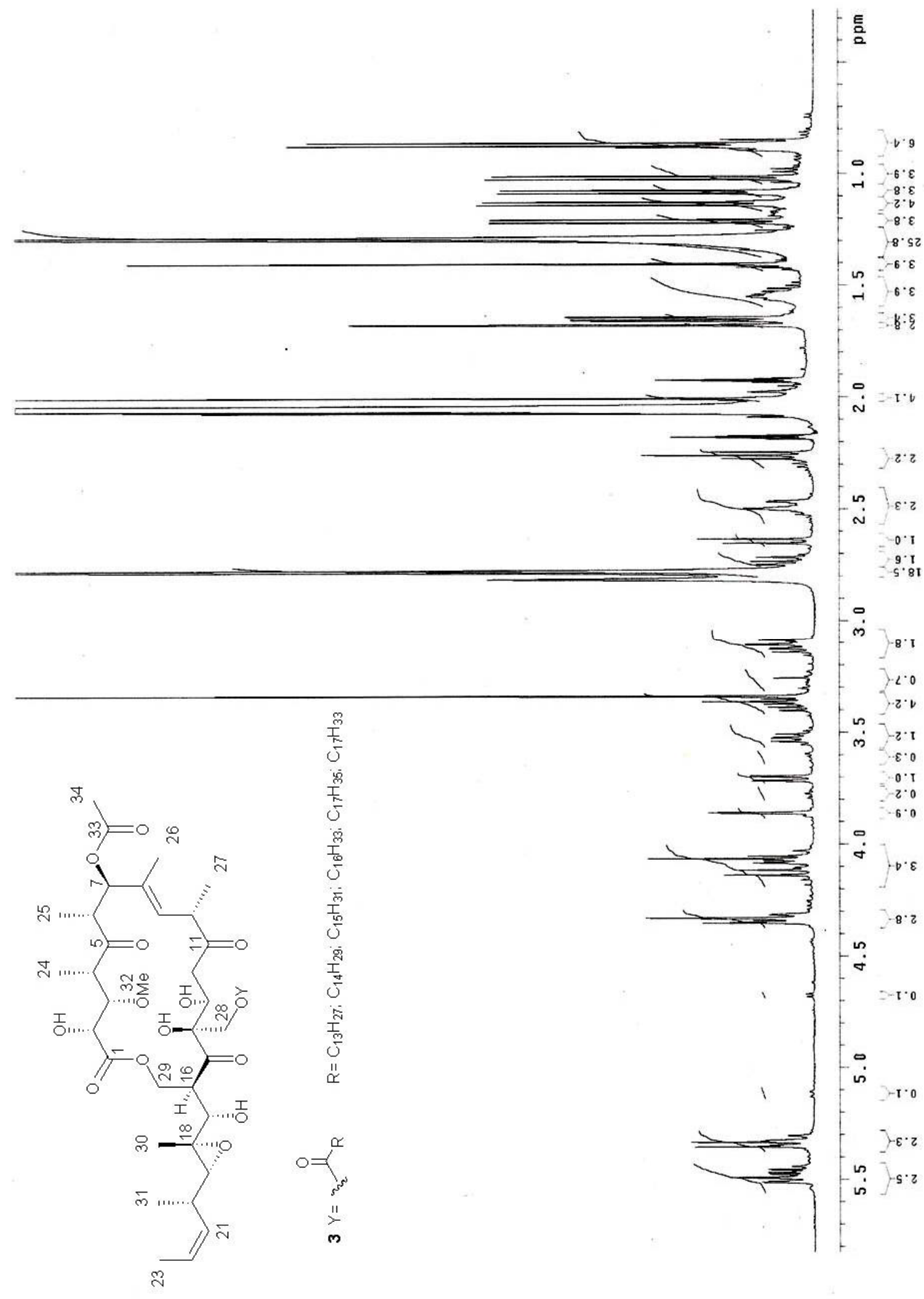

Figure S 2. ${ }^{1} \mathrm{H}$ NMR spectrum candidaspongiolides (3) in acetone- $d_{6}$ 


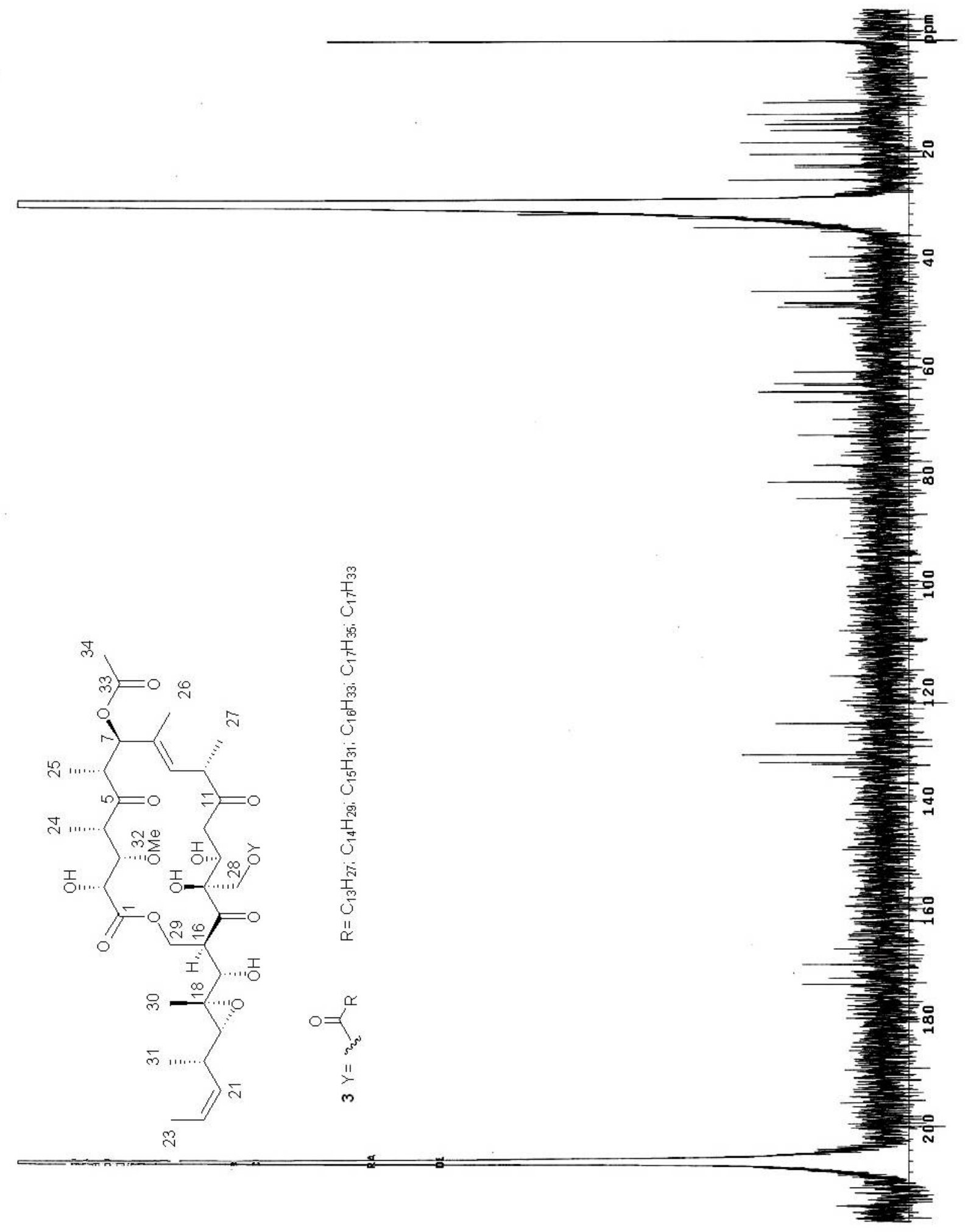

Figure S 3. ${ }^{13} \mathrm{C}$ NMR spectrum candidaspongiolides (3) in acetone- $d_{6}$ S 6 


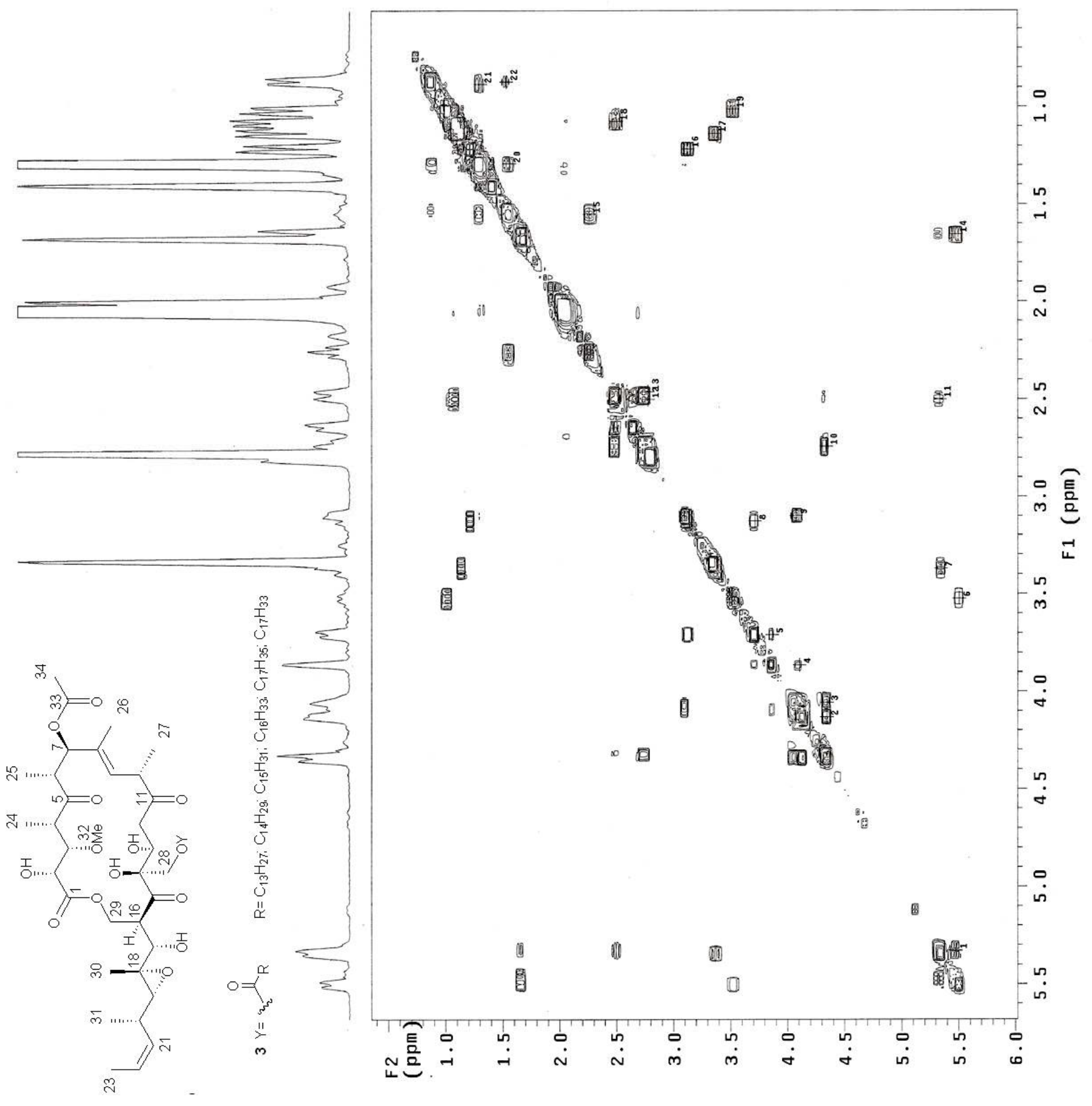

Figure S 4. COSY spectrum candidaspongiolides (3) in acetone- $d_{6}$ 


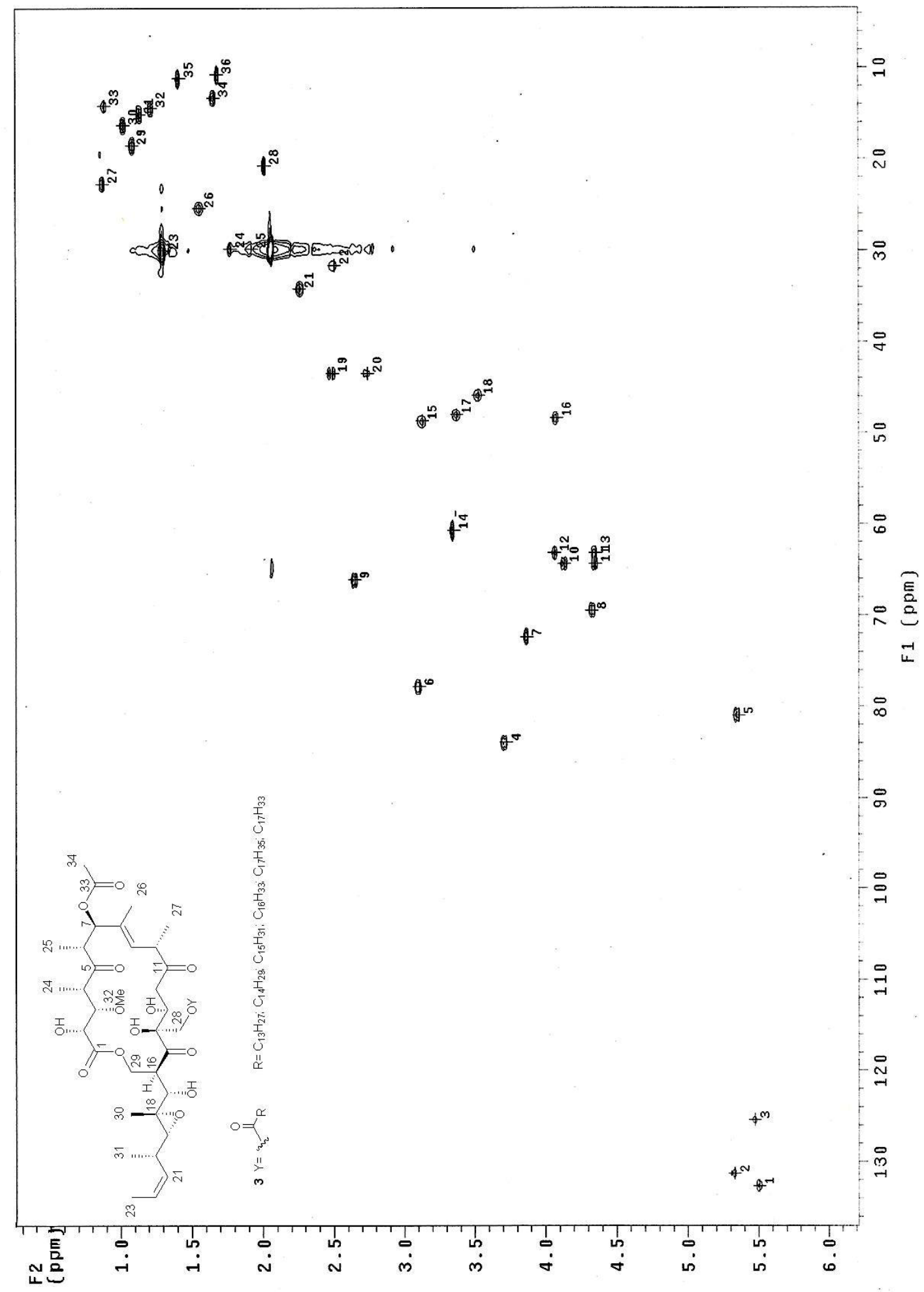

Figure S 5. HSQC spectrum candidaspongiolides (3) in acetone- $d_{6}$ 


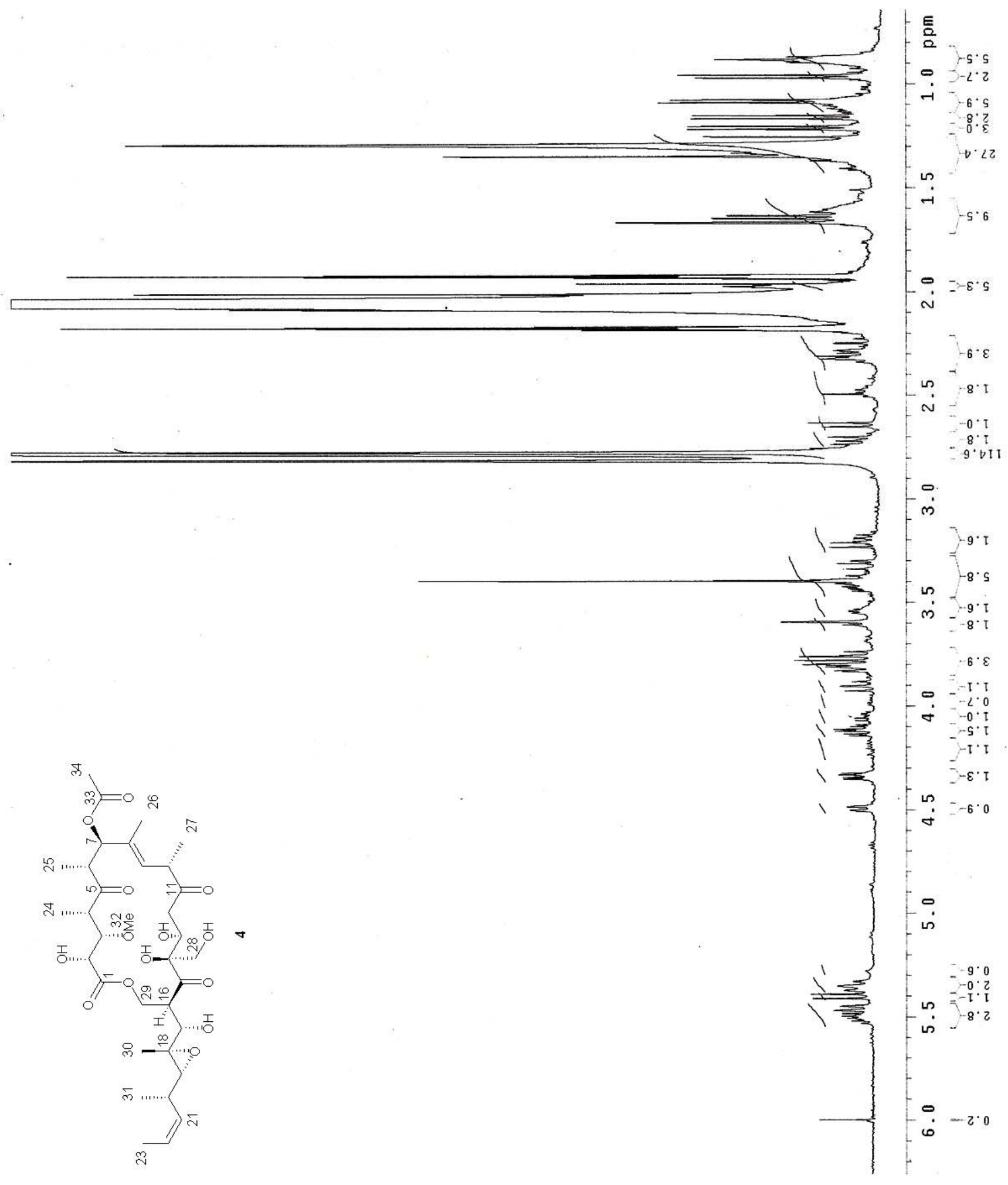

Figure S 6. ${ }^{1} \mathrm{H}$ NMR spectrum candidaspongiolide core (4) in acetone- $d_{6}$ 


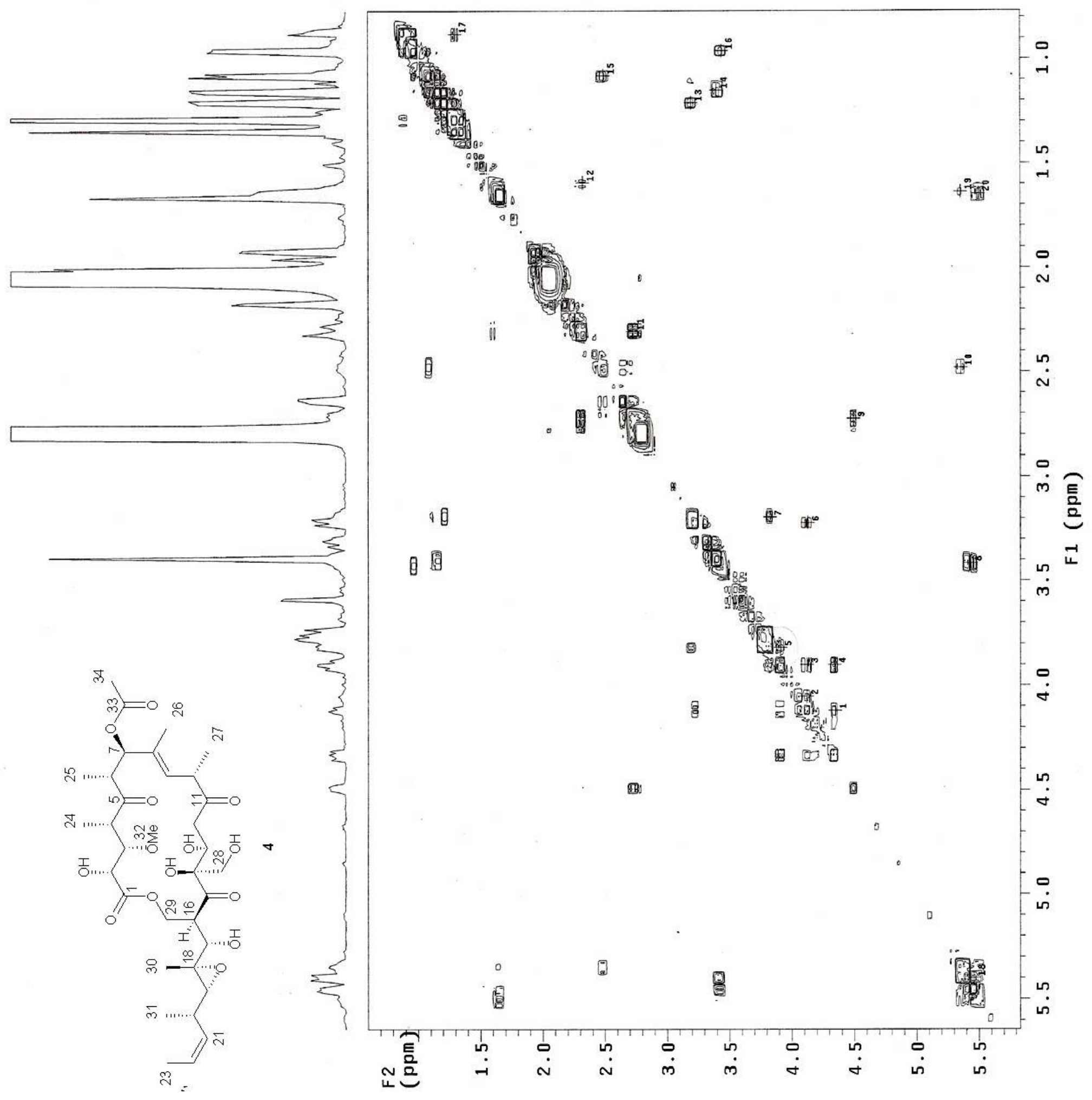

Figure S 7. COSY spectrum candidaspongiolide core (4) in acetone- $d_{6}$ S 10 


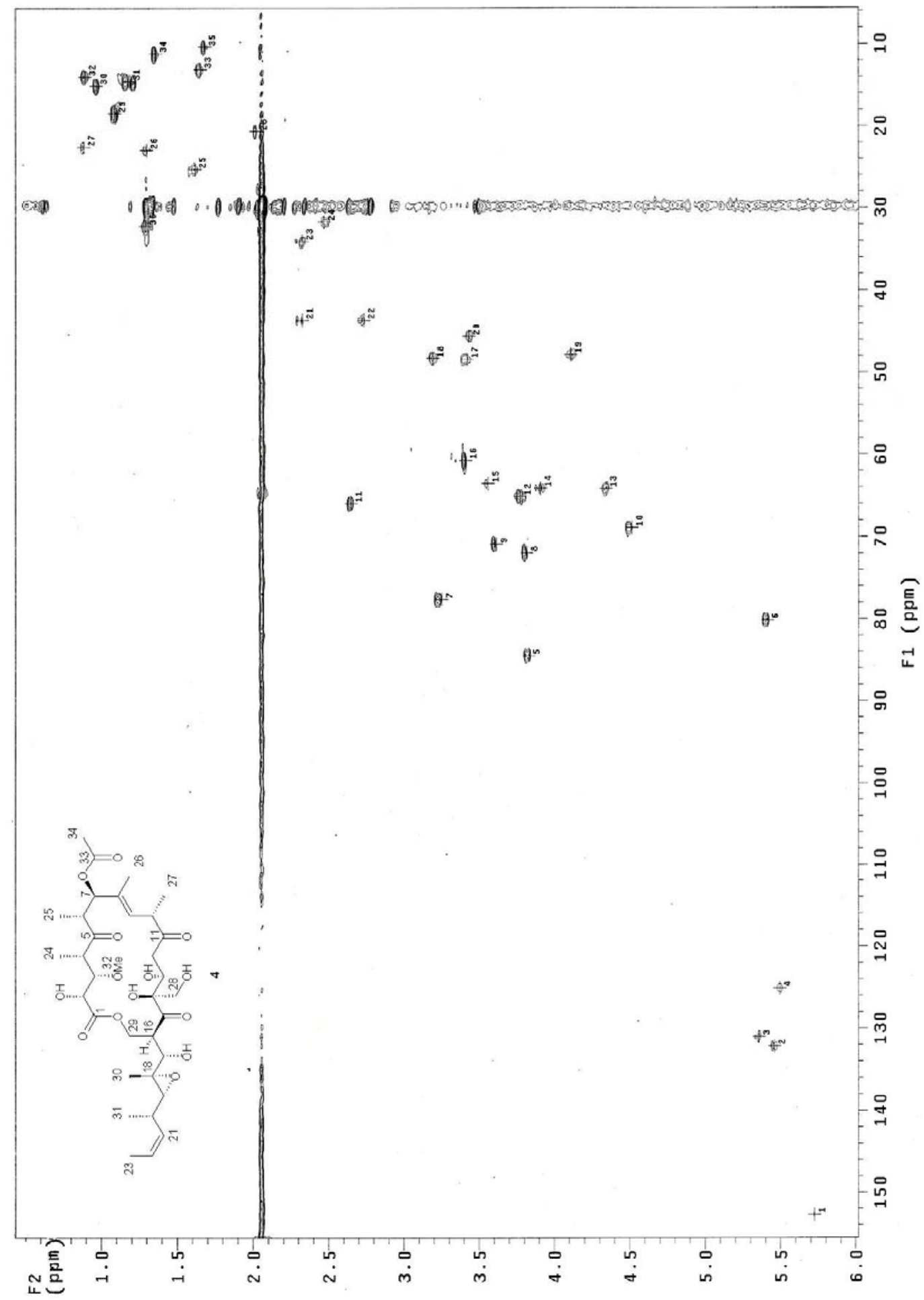

Figure S 8. HSQC spectrum candidaspongiolide core (4) in acetone- $d_{6}$ 


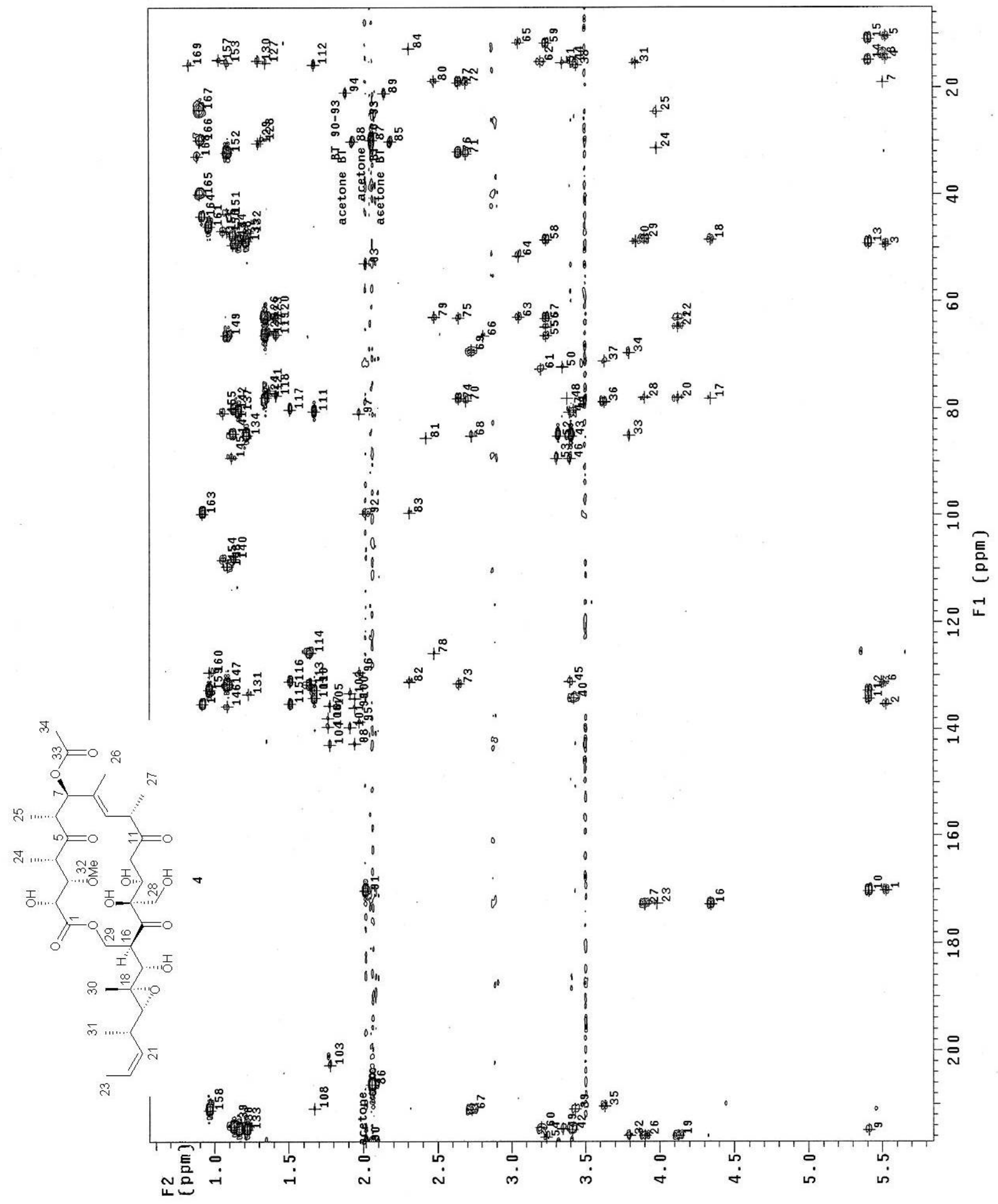

Figure S 9. HMBC (4 Hz) spectrum candidaspongiolide core (4) in acetone- $d_{6}$ S 12 


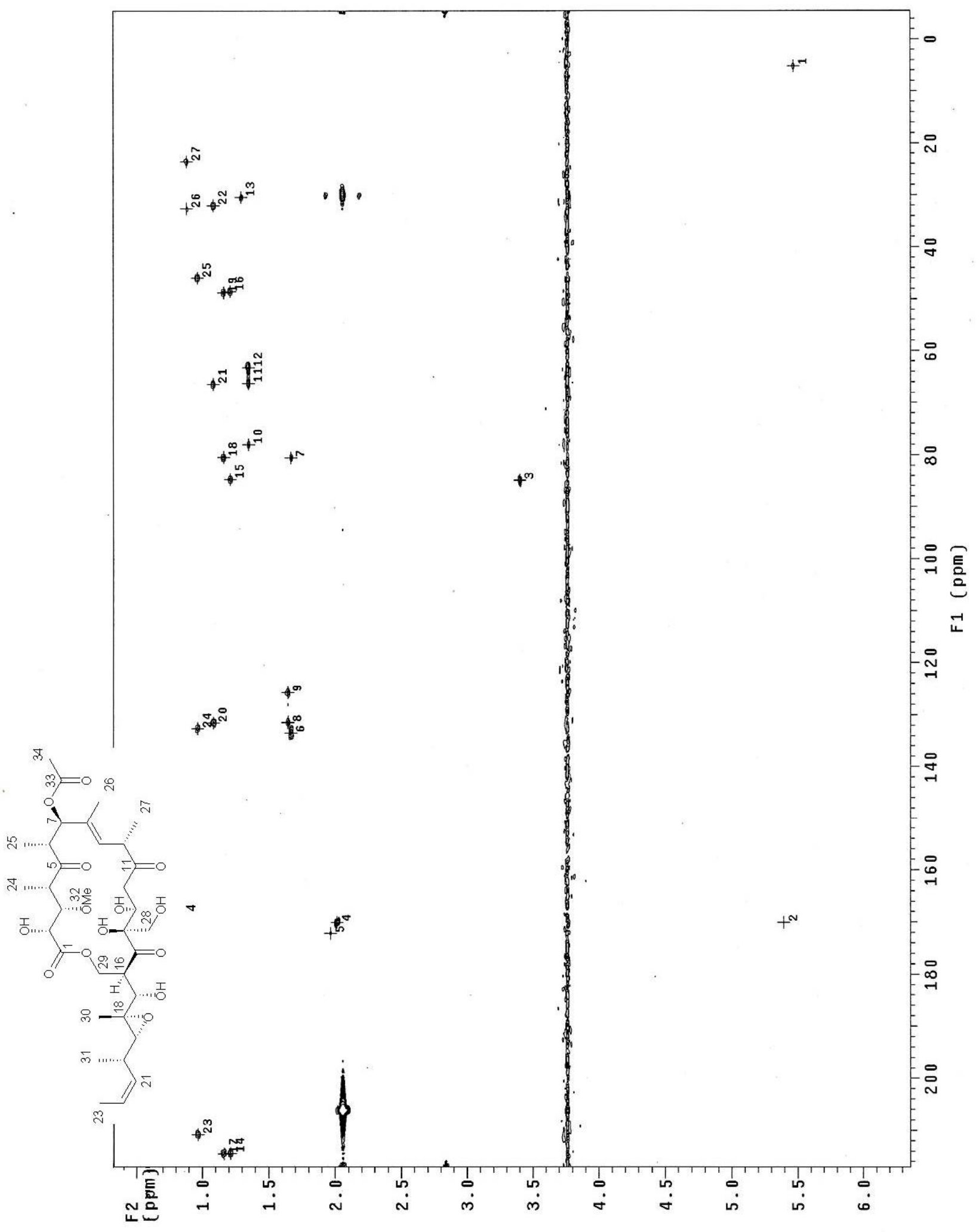

Figure S 10. HMBC ( $8 \mathrm{~Hz})$ spectrum candidaspongiolide core (4) in acetone- $d_{6}$ S 13 


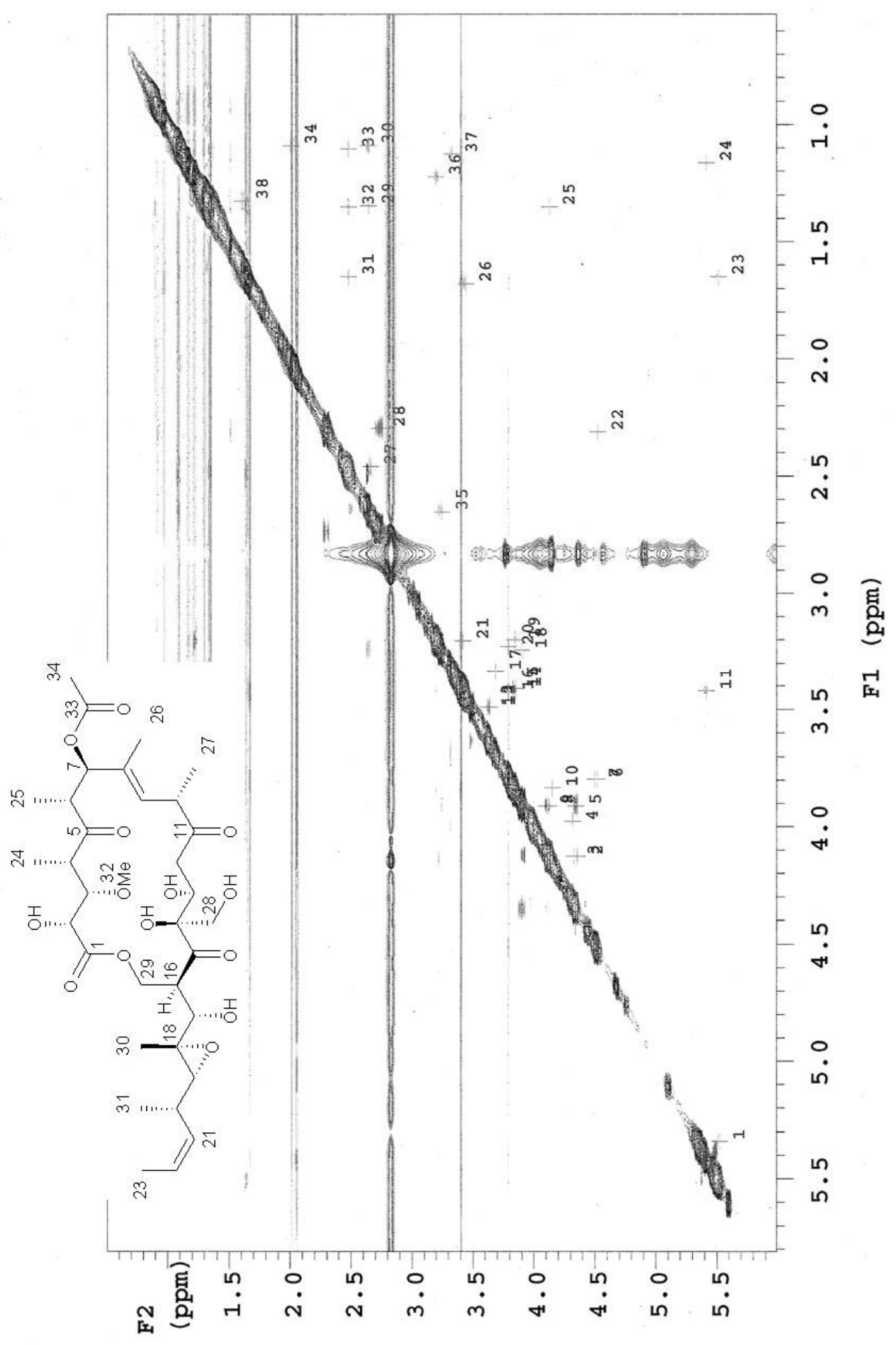

Figure S 11. NOESY spectrum candidaspongiolide core (4) in acetone- $d_{6}$ S 14 